International Journal of Foundations of Computer Science, Vol. 13, No. 6 (2002) 937-940

(C) World Scientific Publishing Company

\title{
AUTHOR INDEX \\ VOLUME 13 (2002)
}

Aichholzer O., Alboul L. S. and Hurtado F., On flips in polyhedral surfaces

13 (2002) 303-312

Alboul L. S., see Aichholzer O.,

Anceaume E., Efficient solution to uniform atomic broadcast

Anceaume E., Helary J.-M. and Raynal M., A note on the determination of the immediate predecessors in a distributed computation

Arslan A. N. and Eğecioğlu Ö., Approximation algorithms for local alignment with length constraints

13 (2002) $303-312$

13 (2002) 695-718

Bajaj C. L., see Xu G.,

Bergeron A. and Hamel S., Vector algorithms for approximate string matching

Bilbao J. M., Fernández J. R. and López J. J., On the complexity of computing values of restricted games

Bodlaender H. L., see Zantema H.,

Brüggemann-Klein A. and Wood D., The regularity of two-way nondeterministic tree automata languages

Câmpeanu C., Salomaa K. and Vágvölgyi S., Shuffle decompositions of regular languages

Cai M. C., see Yang X. G.,

Cameron H., see Mantler A.,

Campeanu C., Paun A. and Yu S., An efficient algorithm for constructing minimal cover automata for finite languages

Cercone N., see Ling C. X.,

Champarnaud J.-M., Evaluation of three implicit structures to implement nondeterministic automata from regular expressions

Cheng E. Y. C. and Sahni S., Gate resizing to reduce power consumption

Cheng S.-W. and Dey T., Preface

Culik II K., Karhumäki J. and Kari J., A note on synchronized automata and road coloring problem

$13(2002) 865-872$

$13(2002) 751-768$

13 (2002) 261-284

$13(2002) 53-66$

$13(2002) 633-652$

13 (2002) 445-458

13 (2002) 67-82

$13(2002) 799-816$

13 (2002) 671-680

13 (2002) 837-863

13 (2002) 83-98

13 (2002) 473-476

13 (2002) 99-114

13 (2002) 405-430

13 (2002) 161-162

13 (2002) 459-472

Dang Z. and Ibarra O. H., The Existence of $\omega$-chains for transitive mixed linear relations and its applications

13 (2002) 911-936

Deng X. T., Algorithmic issues in mathematical economics, Preface

Deng X. T., Li Z.-F. and Wang S.-Y., Computational complexity of arbitrage in frictional security market

$13(2002) 629632$

$13(2002) 681-684$

Deng X.-T., Feng H.-D., Li G.-J. and Liu G.-Z., A PTAS for minimizing total completion time of bounded batch scheduling

Devillers O., Pion S. and Teillaud M., Walking in a triangulation

$13(2002) 817-827$

13 (2002) 181-200 
Devillers O., The Delaunay hierarchy

$13(2002) 163-180$

Dey T., see Cheng S.-W.

$13(2002) 161-162$

Ĕgecioğlu Ö., see Arslan A. N.,

13 (2002) $751-768$

Edelsbrunner H. and Guoy D., Sink insertion for mesh improvement

Estivill-Castro V. and Yang J., Clustering web visitors by fast, robust and convergent algorithms

13 (2002) 223-242

Evans S., see Xu G.,

Faloutsos M., Pankaj R. and Sevcik K. C., The effect of asymmetry on the on-line multicast routing problem

$13(2002) 497-520$

13 (2002) 261-284

$13(2002) 889-910$

Fang Q. and Zhu S., Linear and integer programming techniques for cooperative games

Feng H.-D., see Deng X.-T.,

Fernández J. R., see Bilbao J. M.

Francesco N. D. and Santone A., A formula-driven modular attack on state explosion

Frey W. H., Boundary triangulations approximating developable surfaces that interpolate a close space curve

Fussell D., see Xu W.,

Gao W., Wang S. and Liu B., A dynamic recommendation system based on log mining

Gu Y. G., see Shu J. W.,

Guoy D., see Edelsbrunner H.,

Hamel S., see Bergeron A.,

Hammersley R., see Xu W.,

Harel D. and Kugler H., Synthesizing state-based object systems from LSC specifications

Hartog J. I. D. and Vink E. P. D., Verifying probabilistic programs using a hoare like logic

Helary J.-M., see Anceaume E.,

Henriksen J. G., An expressive extension of TLC

Ho M. K., see $\mathrm{Ng} \mathrm{V}$.,

Honkala J., Remarks concerning the D0L w-equivalence problem,

Hurtado F., see Aichholzer O.,

Hutter M., The fastest and shortest algorithm for all well-defined problems

Ibarra O. H., see Dang Z.,

Ibarra O. H., Verification in queue-connected multicounter machines

Jia W. and Sun Z., On computational complexity of hierarchical optimization

Kókai I., see Lörincz A.,

Kamareddine F. and Monin F., An extension of an automated termination method of recursive functions

Karhumäki J., see Culik II K.,

Kari J., see Culik II K.,

Klarlund N., Møller A. and Schwatzbach M. I., MONA implementation secrets

Kugler H., see Harel D.,

Kumar K. N., see Roychoudhury A.,

$13(2002) 653-666$

$13(2002) 817-827$

$13(2002) 633-652$

$13(2002) 719-732$

13 (2002) 285-302

13 (2002) 243-260

$13(2002) 521-530$

13 (2002) 685-694

13 (2002) 223-242

$13(2002) 53-66$

13 (2002) 243-260

$13(2002) 5-52$

13 (2002) 315-340

$13(2002) 865-872$

13 (2002) 341-360

13 (2002) 531-554

13 (2002) 769-778

13 (2002) 303-312

13 (2002) 431-444

$13(2002)$ 911-936

$13(2002)$ 115-128

13 (2002) 667-670

$13(2002) 477-496$

13 (2002) 361-386

13 (2002) 459-472

13 (2002) 459-472

$13(2002) 571-586$

$13(2002) 5-52$

$13(2002) 387-404$

Lörincz A., Kókai I. and Meretei A., Intelligent

high-performance crawlers used to reveal topic-specific

structure of WWW

$13(2002) 477-496$ 
López J. J., see Bilbao J. M.

Lepère R., Trystram D. and Woeginger G., Approximation algorithms for scheduling malleable tasks under precedence constraints

13 (2002) 613-628

Li G.-J., see Deng X.-T.,

13 (2002) 817-827

Li Z.-F., see Deng X. T.,

$13(2002) 681-684$

Ling C. X. and Cercone N., Editorial notes

$13(2002) 473-476$

Liu B., see Gao W.,

Liu G.-Z., see Deng X.-T.,

13 (2002) 521-530

$13(2002) 817-827$

Liu R. J., see Yang X. G.,

13 (2002) 671-680

Lu K., see Xu W.,

$13(2002) 243-260$

Møller A., see Klarlund N.

Mantler A. and Cameron H., Constructing red-black tree shapes

13 (2002) $571-586$

$13(2002) 837-863$

Martín-Vide C., Mateescu A. and Mitrana V., Parallel finite automata systems communicating by states

13 (2002) 733-750

Mateescu A., see Martín-Vide C.,

Meretei A., see Lörincz A.,

Mitrana V., see Martín-Vide C.,

Mohri M., Generic $\epsilon$-removal and input $\epsilon$-normalization algorithms for weighted transducers

Monin F., see Kamareddine F.

Mourelle L. D. M., see Nedjah N.

N. Zhong, Representation and construction of ontologies for web intelligence

13 (2002) $733-750$

$13(2002) 477-496$

13 (2002) $733-750$

13 (2002) 129-144

$13(2002) 361-386$

13 (2002) 873-887

$13(2002) 555-570$

Nedjah N. and Mourelle L. D. M., Pattern matching code minimization in rewriting-based programming languages

$13(2002) 873-887$

$\mathrm{Ng} \mathrm{V}$. and Ho M. K., An intelligent agent for web advertisements

$13(2002) 531-554$

Păun G., see Păun A.,

Păun A., Păun G. and Rozenberg G., Computing by communication in networks of membranes

$13(2002) 779-798$

Pankaj R., see Faloutsos M.,

13 (2002) $779-798$

$13(2002) 889-910$

$13(2002) 83-98$

Pighizzini G. and Shallit J., Unary language operations, state complexity and Jacobsthal's function

13 (2002) $145-160$

$13(2002) 181-200$

$13(2002) 387-404$

13 (2002) 387-404

$13(2002) 865-872$

Raynal M., see Anceaume E.,

Roychoudhury A., Kumar K. N., Ramakrishnan C. R. and Ramakrishnan I. V., Beyond Tamaki-Sato style unfold/fold transformations for normal logic programs

13 (2002) 387-404

13 (2002) $779-798$

Rozenberg G., see Păun A.,

Sahni S.,

Salomaa K., see Câmpeanu C.,

Santone A., see Francesco N. D.,

$13(2002) 405-430$

$13(2002) 799-816$

$13(2002) 719-732$

Schmidhuber J., Hierarchies of generalized Kolmogorov complexities and nonenumerable universal measures computable in the limit

Schwatzbach M. I., see Klarlund N., 
Sevcik K. C., see Faloutsos M.,

13 (2002) 889-910

Shallit J., see Pighizzini G.

13 (2002) $145-160$

Sheffer A., see Üngör A.,

13 (2002) 201-222

Shu J. W., Gu Y. G. and Zheng W. M., A novel numerical approach of computing American option

13 (2002) 685-694

Sun Z., see Jia W.

Tao S., see Yang X. G.,

$13(2002) 667-670$

13 (2002) 671-680

Teillaud M., see Devillers O.,

Thiagarajan P. S. and Yap R., Preface

13 (2002) 181-200

$13(2002) 313-314$

$13(2002) 829-835$

Tran N., On universally polynomial context-free languages

13 (2002) 613-628

Üngör A. and Sheffer A., Pitching tents in space-time:

Mesh generation for discontinuous Galerkin method

13 (2002) 201-222

$13(2002) 799-816$

13 (2002) 315-340

13 (2002) 521-530

$13(2002) 681-684$

$13(2002) 613-628$

$13(2002) 67-82$

Wood D., see Brüggemann-Klein A.

Xu G., Bajaj C. L. and Evans S., $C^{1}$ modeling with hybrid multiple-sided a-patches

$13(2002)$ 261-284

Xu W., Hammersley R., Lu K. and Fussell D., Lossless subdivision-based multiresolution representation of arbitrary triangle meshes using kite trees

$13(2002) 243-260$

$13(2002) 497-520$

Yang X. G., Tao S., Liu R. J. and Cai M. C., Complexity of scenario-based portfolio optimization problem with VaR objective

Yap R., see Thiagarajan P. S.,

$13(2002) 671-680$

$13(2002) 313-314$

$13(2002) 83-98$

Yu S., Implementation and application of automata, Editor's Foreword

$13(2002) 1-4$

$13(2002) 445-458$

$13(2002) 685-694$

13 (2002) 653-666 